\title{
Pattern formation simulations in reaction-diffusion systems by local integral equation method
}

\author{
V. Sladek ${ }^{1}$, J. Sladek ${ }^{1} \&$ A. Shirzadi ${ }^{2}$ \\ ${ }^{1}$ Institute of Construction and Architecture, Slovak Academy of Sciences, \\ Bratislava, Slovakia \\ ${ }^{2}$ Department of Mathematics, Persian Gulf University, Bushehr, Iran
}

\begin{abstract}
A meshless Local Integral Equation (LIE) method is proposed for numerical simulation of 2D pattern formation in nonlinear reaction-diffusion systems. The method works with weak formulation of the differential governing equations on local sub-domains with using the Green function of the Laplace operator as the test function. The Moving Least Square (MLS) approximation is employed for spatial variations of field variables while the time evolution is discretized by using suitable finite difference approximations. The effects of model parameters and conditions are studied by considering the well known Schnakenberg model. Keywords: nonlinear reaction-diffusion systems, Turing instability, pattern formation, Schnakenberg model, meshless methods, local integral equations, moving least squares, finite differences.
\end{abstract}

\section{Introduction}

Alan Turing demonstrated [1] how a simple model system of coupled reactiondiffusion equations could give rise to spatial patterns in chemical concentrations through a process of chemical instability (diffusion driven instability). Turing also pointed out the role of such patterns in biological pattern formation. A large variety of pattern formation with various applications has been explained by Turing type models. It is not the aim of this paper to give a review of such application and modelling of pattern formation [2]. Recall that several Turing models have been developed such as the Gierer-Meinhardt model [3], Gray-Scott model [4], Lengyel-Epstein model [5], Brusselator model [6], Schnakenberg 
model [7] and Selkov model [8]. A typical Turing system is a reaction-diffusion system consisting of at least two chemical species (activator and inhibitor) exhibiting a steady state which is stable to small perturbations in the absence of diffusion, but becomes unstable when diffusion is present (Turing instability). The formation of spatial patterns is principally a nonlinear phenomenon. Otherwise the unstable modes would grow unlimitedly. Linear theory does determine conditions under which spontaneous pattern formation is allowed for certain parameter ranges [2]. Initial and boundary conditions, the shape and size of the domain yield various forms of patterns. To determine which of the various possible patterns will be stable or which conversion takes place, one has to go beyond linear theory. Various numerical methods have been used to solution of nonlinear reaction-diffusion systems and computer simulations of pattern formation.

In this paper, we develop the Local integral equation (LIE) method for numerical simulations of 2-d pattern formation in reaction-diffusion systems. The method is truly meshless, since no elements are required either for approximations or for integration in the analysed domain. The Moving Least Square (MLS) approximation is employed for spatial variations of field variables while the time evolution is discretized by using one-step $\theta$-method. The nodal points are distributed freely inside the analysed domain and on its boundary without using any connectivity among nodes. The LIE is a weak formulation of the differential governing equations on local sub-domains considered around each interior node with using the Green function of the Laplace operator as the test function. Thus the integral form of the governing equations is the integral representation of field variables at interior nodes. The appropriate choice of the shape of sub-domains enables us to find the Green function vanishing on the boundary of the sub-domain and so eliminate the normal derivatives of the field variables from the formulation. This is valuable achievement since the accuracy of approximations for derivatives is lower than for primary fields and also the evaluation of derivatives at integration points prolongs the computation. The nonlinear terms are treated iteratively within each time step. Several numerical simulations are presented in order to verify the developed method. Attention is paid to illustrate the dependence of pattern shape on the initial conditions, size and shape of the analysed domain.

\section{Mathematical formulation of reaction-diffusion problems}

The governing equations for the concentrations of two chemicals $u(\mathbf{x}, t)$ and $v(\mathbf{x}, t)$ subjected to reaction-diffusion processes are given as [2]

$$
\frac{\partial u}{\partial t}=\nabla^{2} u+\gamma f(u, v), \quad \frac{\partial v}{\partial t}=d \nabla^{2} v+\gamma g(u, v) \quad \text { in } \Omega \times[0, T]
$$


These equations must be completed with prescription of the initial values $\{u(\mathbf{x}, 0), v(\mathbf{x}, 0)\}$ and the boundary conditions, which are usually taken as the Neumann type

$$
\frac{\partial u}{\partial \mathbf{n}}=0, \frac{\partial v}{\partial \mathbf{n}}=0 \text { on } \partial \Omega
$$

The zero flux conditions imply no external input. If we imposed fixed boundary conditions on $u$ and $v$, the spatial patterning could be a direct consequence of the boundary conditions (as it can be seen in ecological problems).

The relevant homogeneous steady state $\left(u_{0}, v_{0}\right)$ of $(1)$ is the positive solution of

$$
f(u, v)=0, \quad g(u, v)=0 .
$$

Since we are concerned with diffusion-driven instability, the steady state must be homogeneous and satisfy the equations

$$
\frac{\partial u}{\partial t}=\gamma f(u, v), \quad \frac{\partial v}{\partial t}=\gamma g(u, v),
$$

Hence, assuming linear stability for $w=\left(u-u_{0}, v-v_{0}\right)^{T}$ about the steady state $\left(u_{0}, v_{0}\right)^{T}$, we obtain the governing equations

$$
\frac{\partial w}{\partial t}=\gamma A w, \quad A=\left(\begin{array}{ll}
f_{, u} & f_{, v} \\
g_{, u} & g_{, v}
\end{array}\right),
$$

with the partial derivatives of $f$ and $g$ being evaluated at the steady state.

Looking for the time evolution in the form $w \sim e^{\omega t}$, where $\omega$ is the eigenvalue, we get the condition $\operatorname{Re} \omega<0$ for requirement of linear stability of the steady state $w=0$. It can be shown [2] that the linear stability is guaranteed if

$$
f_{, u}+g_{, v}<0, \quad f_{, u} g_{, v}-f_{, v} g_{, u}>0 .
$$

The solution of the linearized full reaction-diffusion system (1),

$$
\frac{\partial w}{\partial t}=D \nabla^{2} w+\gamma A w, \quad D=\left(\begin{array}{ll}
1 & 0 \\
0 & d
\end{array}\right),
$$

with using the expansion in terms of the eigenfunctions of the Laplace operator corresponding to eigenvalues $k^{2}$, yields the dispersion relation $\omega\left(k^{2}\right)$. An analysis of the dispersion relation is extremely informative in that it immediately says which eigenfunctions, i.e. which spatial patterns are linearly unstable and grow exponentially with time. Note that with finite domain eigenvalue problems, 
the wavenumbers are discrete and so only certain $k$ are relevant. Of course in nonlinear RD problems there are mechanisms which do not allow unbounded growth of unstable modes with $t \rightarrow \infty$. Therefore the linear stability analysis (though informative) is not complete for post buckling evolution and it is necessary to know the solution of the nonlinear problems with taking into account the prescribed boundary and initial conditions as well as the geometry of the analysed domain.

Nevertheless, the linear stability analysis yields necessary conditions for the generation of spatial patterns. In the case of two species RD mechanisms of the form (1) these conditions can be summarized as [2]

$$
\begin{array}{ll}
f_{, u}+g_{, v}<0, & \left(f_{, u} g_{, v}-f_{, v} g_{, u}\right)>0 \\
\left(d f_{, u}+g_{, v}\right)>0, & \left(d f_{, u}+g_{, v}\right)^{2}-4 d\left(f_{, u} g_{, v}-f_{, v} g_{, u}\right)>0 .
\end{array}
$$

Being satisfied such conditions, the formation of spatial patterns and their shape depends on the boundary and initial conditions as well as on the shape of the domain.

\section{Local integral equation formulation and numerical implementation}

The governing equations (1) should be valid on an arbitrary sub-domain $\Omega^{s}$ of the analysed 2-d domain $\Omega$. The weak form of these equations on a local subdomain (the local weak form) is given as

$$
\int_{\Omega^{s}}\left[\frac{\partial u}{\partial t}-\nabla^{2} u-\gamma f(u, v)\right] u^{*} d \mathbf{x}=0, \int_{\Omega^{s}}\left[\frac{\partial v}{\partial t}-d \nabla^{2} v-\gamma g(u, v)\right] u^{*} d \mathbf{x}=0
$$

where $u^{*}$ is a test function. Basically, the test function can be selected arbitrarily. One of the convenient choices is to take it as the Green function of the Laplace operator with vanishing on the boundaries of appropriately chosen sub-domains.

Let the sub-domain $\Omega^{c}$ be a circle of the radius $r_{0}$ and centred at the point $\mathbf{x}^{c} \in \Omega$. Then,

$$
u^{*}(r)=-\frac{1}{2 \pi} \ln \left(\frac{r}{r_{0}}\right), \quad r=\|\mathbf{r}\|=\left\|\mathbf{x}-\mathbf{x}^{c}\right\|
$$

obeys the equations

$$
\nabla^{2} u^{*}(r)=-\delta(\mathbf{r}),\left.\quad u^{*}(r)\right|_{\partial \Omega^{c}}=0 .
$$

In view of the Gauss divergence theorem, Eqs. (8)-(10) can be rewritten as 


$$
\begin{gathered}
u\left(\mathbf{x}^{c}, t\right)+\int_{\partial \Omega^{c}} u(\mathbf{x}, t) \frac{\partial u^{*}\left(\left\|\mathbf{x}-\mathbf{x}^{c}\right\|\right)}{\partial \mathbf{n}(\mathbf{x})} d \Gamma+ \\
+\int_{\Omega^{c}}\left[\frac{\partial u(\mathbf{x}, t)}{\partial t}-\gamma f(u(\mathbf{x}, t), v(\mathbf{x}, t))\right] u^{*}\left(\left\|\mathbf{x}-\mathbf{x}^{c}\right\|\right) d \Omega=0 \\
d v\left(\mathbf{x}^{c}, t\right)+d \int_{\partial \Omega^{c}} v(\mathbf{x}, t) \frac{\partial u^{*}\left(\left\|\mathbf{x}-\mathbf{x}^{c}\right\|\right)}{\partial \mathbf{n}(\mathbf{x})} d \Gamma+ \\
+\int_{\Omega^{c}}\left[\frac{\partial v(\mathbf{x}, t)}{\partial t}-\gamma g(u(\mathbf{x}, t), v(\mathbf{x}, t))\right] u^{*}\left(\left\|\mathbf{x}-\mathbf{x}^{c}\right\|\right) d \Omega=0 .
\end{gathered}
$$

Recall that the derived LIEs do not involve normal derivatives of the field variables $u(\mathbf{x}, t)$ and $v(\mathbf{x}, t)$ on the boundary $\partial \Omega^{c}$.

Besides adopting the local weak formulation, we employ the moving leastsquares (MLS) approximation [9] for the field variable $w(\mathbf{x}, t) \in\{u(\mathbf{x}, t), v(\mathbf{x}, t)\}$ in order to receive completely mesh-free formulation.

Then,

$$
w(\mathbf{x}, t) \approx \tilde{w}(\mathbf{x}, t):=\sum_{a=1}^{n} \hat{w}^{a}(t) \phi^{a}(\mathbf{x}) \quad,
$$

where $\phi^{a}(\mathbf{x})$ is the shape function associated with the node $\mathbf{x}^{a}$ and the expansion coefficient $\hat{w}^{a}(t)$ is the nodal unknown which is different from the nodal value $w\left(\mathbf{x}^{a}, t\right)$, in general. Note that the nodal points are spread freely in $(\Omega \cup \partial \Omega)$ without any connectivity among the nodes. The derivatives of the field variables can be approximated as derivatives of (12). Recall that the evaluation of the shape function at each point requires a computational procedure which prolong the computational time. The computational procedure is becoming more time consuming with increasing the order of the derivatives of the shape functions. Moreover, the accuracy of the derivatives is decreasing with increasing their order [10]. Fortunately, in the present formulation the derivatives of the field variables are reduced to the $1^{\text {st }}$ order and they are evaluated only at the nodal points instead of the integration points.

Collocation of the LIEs (11) at interior nodes $\mathbf{x}^{c} \in \Omega$, while the prescribed boundary conditions (2) at boundary nodes $\mathbf{x}^{b} \in \partial \Omega$, with using the MLSapproximations for field variables and their derivatives, we obtain the system of the ordinary differential equations for unknowns $\left\{\hat{u}^{a}(t), \hat{v}^{a}(t)\right\},(a=1,2, \ldots, n)$

$$
\sum_{a=1}^{n} \frac{\partial \hat{u}^{a}(t)}{\partial t} A^{c a}+\sum_{a=1}^{n} \hat{u}^{a}(t) B^{c a}-\gamma \int_{\Omega^{c}} f(\tilde{u}(\mathbf{x}, t), \tilde{v}(\mathbf{x}, t)) u^{*}\left(\left\|\mathbf{x}-\mathbf{x}^{c}\right\|\right) d \Omega=0
$$




$$
\begin{gathered}
\sum_{a=1}^{n} \frac{\partial \hat{v}^{a}(t)}{\partial t} A^{c a}+d \sum_{a=1}^{n} \hat{v}^{a}(t) B^{c a}-\gamma \int_{\Omega^{c}} g(\tilde{u}(\mathbf{x}, t), \tilde{v}(\mathbf{x}, t)) u^{*}\left(\left\|\mathbf{x}-\mathbf{x}^{c}\right\|\right) d \Omega=0\left(13 b \quad \text { at } \mathbf{x}^{b} \in \partial \Omega\right. \\
\sum_{a=1}^{n} \hat{u}^{a}(t) D^{b a}=0, \quad \sum_{a=1}^{n} \hat{v}^{a}(t) D^{b a}=0, \quad
\end{gathered}
$$

where

$$
\begin{gathered}
A^{c a}=\int_{\Omega^{c}} \phi^{a}(\mathbf{x}) u^{*}\left(\left\|\mathbf{x}-\mathbf{x}^{c}\right\|\right) d \Omega, \\
B^{c a}=\phi^{a}\left(\mathbf{x}^{c}\right)+\int_{\partial \Omega^{c}} \phi^{a}(\mathbf{x}) \frac{\partial u^{*}\left(\left\|\mathbf{x}-\mathbf{x}^{c}\right\|\right)}{\partial \mathbf{n}(\mathbf{x})} d \Gamma, \quad D^{b a}=\frac{\partial \phi^{a}}{\partial \mathbf{n}}\left(\mathbf{x}^{b}\right) .
\end{gathered}
$$

Recall that the last integral terms in (13) involve a non-linearity depending on the kinetics of the considered reaction-diffusion system.

\subsection{Approximate integrations}

The shape of the sub-domains is arbitrary. In order to facilitate the integrations involved in the LIEs, we shall consider circular sub-domains centred at interior nodes and with radius $r_{0}$. Then, the integration over $\partial \Omega^{c}$ is reduced to the angular integration which can be performed by using the standard GaussLegendre quadrature rule as

$$
\begin{gathered}
C^{c a}:=\int_{\partial \Omega^{c}} \phi^{a}(\mathbf{x}) \frac{\partial u^{*}\left(\left\|\mathbf{x}-\mathbf{x}^{c}\right\|\right)}{\partial \mathbf{n}(\mathbf{x})} d \Gamma=-\frac{1}{2 \pi} \int_{0}^{2 \pi} \phi^{a}\left(x_{1}^{c}+r_{0} \cos \varphi, x_{2}^{c}+r_{0} \sin \varphi\right) d \varphi= \\
\approx-\frac{1}{2} \sum_{g=1}^{N} w_{g} \phi^{a}\left(x_{1}^{c}+r_{0} \cos \pi\left(1+s_{g}\right), x_{2}^{c}+r_{0} \sin \pi\left(1+s_{g}\right)\right), \\
B^{c a} \approx \phi^{a}\left(\mathbf{x}^{c}\right)+C^{c a},
\end{gathered}
$$

where $w_{g}, s_{g} \in[-1,1]$ are the Gaussian weights and integration points.

The domain integrals can be rearranged as follows

$$
\int_{\Omega^{c}} h(\mathbf{x}) u^{*}\left(\left\|\mathbf{x}-\mathbf{x}^{c}\right\|\right) d \Omega=-h\left(\mathbf{x}^{c}\right) \int_{0}^{r_{0}} r \ln \frac{r}{r_{0}} d r+O\left(r_{0}^{4}\right)=\left(\frac{r_{0}}{2}\right)^{2} h\left(\mathbf{x}^{c}\right)+O\left(r_{0}^{4}\right)
$$

Assuming the radius of sub-domains to be sufficiently small, one can neglect the terms $O\left(r_{0}^{4}\right)$. Thus, 


$$
\begin{gathered}
A^{c a} \approx\left(\frac{r_{0}}{2}\right)^{2} \phi^{a}\left(\mathbf{x}^{c}\right) \\
\int_{\Omega^{c}} f(\tilde{u}(\mathbf{x}, t), \tilde{v}(\mathbf{x}, t)) u^{*}\left(\left\|\mathbf{x}-\mathbf{x}^{c}\right\|\right) d \Omega \approx\left(\frac{r_{0}}{2}\right)^{2} f\left(\tilde{u}\left(\mathbf{x}^{c}, t\right), \tilde{v}\left(\mathbf{x}^{c}, t\right)\right) \\
\int_{\Omega^{c}} g(\tilde{u}(\mathbf{x}, t), \tilde{v}(\mathbf{x}, t)) u^{*}\left(\left\|\mathbf{x}-\mathbf{x}^{c}\right\|\right) d \Omega \approx\left(\frac{r_{0}}{2}\right)^{2} g\left(\tilde{u}\left(\mathbf{x}^{c}, t\right), \tilde{v}\left(\mathbf{x}^{c}, t\right)\right) .
\end{gathered}
$$

Now, the system of the non-linear ODE (13)-(14) becomes

$$
\left(\begin{array}{cc}
\mathbf{M} \frac{\partial}{\partial t}+\mathbf{K} & \mathbf{0} \\
\mathbf{0} & \mathbf{M} \frac{\partial}{\partial t}+d \mathbf{K}
\end{array}\right)\left\{\begin{array}{c}
\hat{\mathbf{U}} \\
\hat{\mathbf{V}}
\end{array}\right\}-\left\{\begin{array}{l}
\mathbf{F} \\
\mathbf{G}
\end{array}\right\}=\left\{\begin{array}{l}
\mathbf{0} \\
\mathbf{0}
\end{array}\right\},
$$

where $\mathbf{M}, \mathbf{K}$ are $(n \times n)$ matrices,

$$
\begin{aligned}
& M^{d a}=\left\{\begin{array}{c}
A^{d a}, \mathbf{x}^{d} \in \Omega \\
0, \quad \mathbf{x}^{d} \in \partial \Omega
\end{array} \quad, \quad K^{d a}=\left\{\begin{array}{c}
B^{d a}, \mathbf{x}^{d} \in \Omega \\
D^{d a}, \mathbf{x}^{d} \in \partial \Omega
\end{array}, \quad(d, a=1,2, \ldots, n)\right.\right. \\
& \hat{\mathbf{U}}=\left\{\hat{u}^{1}(t), \ldots, \hat{u}^{n}(t)\right\}^{T}, \quad \hat{\mathbf{v}}=\left\{\hat{v}^{1}(t), \ldots, \hat{v}^{n}(t)\right\}^{T}, \quad \mathbf{F}=\left\{F^{1}, \ldots, F^{n}\right\}^{T}, \\
& \mathbf{G}=\left\{G^{1}, \ldots, G^{n}\right\}^{T}, \\
& F^{d}=\gamma\left(\frac{r_{0}}{2}\right)^{2}\left\{\begin{array}{ll}
f\left(\tilde{u}\left(\mathbf{x}^{d}, t\right), \tilde{v}\left(\mathbf{x}^{d}, t\right)\right), & \mathbf{x}^{d} \in \Omega \\
0, & \mathbf{x}^{d} \in \partial \Omega
\end{array},\right. \\
& G^{d}=\gamma\left(\frac{r_{0}}{2}\right)^{2}\left\{\begin{array}{ll}
g\left(\tilde{u}\left(\mathbf{x}^{d}, t\right), \tilde{v}\left(\mathbf{x}^{d}, t\right)\right), & \mathbf{x}^{d} \in \Omega \\
0, & \mathbf{x}^{d} \in \partial \Omega
\end{array} .\right.
\end{aligned}
$$

\subsection{Time discretization}

For approximation of the time evolution within the interval $\left[t_{k}, t_{k+1}\right]=\left[t_{k}, t_{k}+\Delta t\right]$, we adopt the $\theta$-method, when

$$
\frac{\hat{w}_{k+1}^{a}-\hat{w}_{k}^{a}}{\Delta t}=\theta \frac{\partial \hat{w}_{k+1}^{a}}{\partial t}+(1-\theta) \frac{\partial \hat{w}_{k}^{a}}{\partial t}, \quad \hat{w}_{k}^{a}=\hat{w}^{a}\left(t_{k}\right) \in\left\{\hat{u}^{a}\left(t_{k}\right), \hat{v}^{a}\left(t_{k}\right)\right\},
$$


Now, in view of (20) and (21), we obtain the system of nonlinear algebraic equations for $2 n$ unknowns $\left\{\hat{u}_{k+1}^{a}, \hat{v}_{k+1}^{a}\right\},(a=1, \ldots, n)$

$$
\begin{gathered}
\left(\begin{array}{cc}
\mathbf{M}+\theta \Delta t \mathbf{K} & \mathbf{0} \\
\mathbf{0} & \mathbf{M}+d \theta \Delta t \mathbf{K}
\end{array}\right)\left\{\begin{array}{c}
\left.\hat{\mathbf{U}}_{k+1}\right\} \\
\hat{\mathbf{V}}_{k+1}
\end{array}\right\}-\theta \Delta t\left\{\begin{array}{c}
\mathbf{F}_{k+1} \\
\mathbf{G}_{k+1}
\end{array}\right\}= \\
=\left(\begin{array}{cc}
\mathbf{M}-(1-\theta) \Delta t \mathbf{K} & \mathbf{0} \\
\mathbf{0} & \mathbf{M}-d(1-\theta) \Delta t \mathbf{K}
\end{array}\right)\left\{\begin{array}{c}
\hat{\mathbf{U}}_{k} \\
\hat{\mathbf{V}}_{k}
\end{array}\right\}+(1-\theta) \Delta t\left\{\begin{array}{c}
\mathbf{F}_{k} \\
\mathbf{G}_{k}
\end{array}\right\},
\end{gathered}
$$

which should be solved subsequently for $(k=0,1, \ldots, F)$. The subscript " $k$ " in the column vectors $\hat{\mathbf{U}}_{k}, \hat{\mathbf{V}}_{k}, \mathbf{F}_{k}, \mathbf{G}_{k}$ means that these vectors are taken at the time instant $t_{k}$. The initial values $\hat{w}_{0}^{a}$ can be obtained from the initial values $w\left(\mathbf{x}^{c}, 0\right)$ by using the approximations

$$
\sum_{a=1}^{n} \hat{w}_{0}^{a} \phi^{a}\left(\mathbf{x}^{c}\right)=w\left(\mathbf{x}^{c}, 0\right), \quad(c=1, \ldots, n) .
$$

The nonlinearities are involved in $\mathbf{F}$ and $\mathbf{G}$ terms and they can be treated iteratively in each time step by replacing $\mathbf{F}_{k+1}$ and $\mathbf{G}_{k+1}$ in the initial iteration by $\mathbf{F}_{k}$ and $\mathbf{G}_{k}$ from the last iteration of the previous time step.

\section{Numerical examples}

Let us consider the Schnakenberg model which is one of the most interesting Turing models in pattern formation. The Schnakenberg model in the dimensionless form is as follows [2]

$$
\frac{\partial u}{\partial t}=\nabla^{2} u+\gamma\left(a-u+u^{2} v\right), \quad \frac{\partial v}{\partial t}=d \nabla^{2} v+\gamma\left(b-u^{2} v\right)
$$

which is a special case of Turing system (1) with $f=a-u+u^{2} v$, $g=b-u^{2} v$, and $\gamma \sim L^{2}$ being scale dependent. The uniform positive steady state $\left(u_{0}, v_{0}\right)$ is

$$
u_{0}=a+b, \quad v_{0}=\frac{b}{(a+b)^{2}}, b>0, a+b>0
$$

and the inequalities (7) results in conditions

$$
\begin{aligned}
& 0<b-a<(a+b)^{3}, \quad(a+b)^{2}>0, \quad d(b-a)>(a+b)^{3} \\
& {\left[d(b-a)-(a+b)^{2}\right]^{2}>4 d(a+b)^{4}}
\end{aligned}
$$


which define a domain in $(a, b, d)$-parameter space, called the pattern formation space (or Turing space), within which the mechanisms is unstable to certain spatial disturbances of wavenumbers $k$ determined by the boundary conditions and falling into the interval $k_{1}^{2}<k^{2}<k_{2}^{2}$ with [2]

$$
k_{1(2)}^{2}=\frac{\gamma}{2 d(a+b)}\left\{d(b-a)-(a+b)^{3} \mp\left[\left(d(b-a)-(a+b)^{3}\right)^{2}-4 d(a+b)^{4}\right]^{1 / 2}\right\}
$$

Since $\gamma \sim L^{2}$ is scale dependent, increasing the size of the domain yields larger limit values of the wave numbers $k_{1}$ and $k_{2}$ which results in new shorter wave lengths of allowable patterns.

In the presented examples, we shall illustrate the influence of the parameters, size and shape of the domain on the pattern formation and the shape of patterns.

In numerical calculations, we have used fixed parameters for the shape parameter of Gaussian weight functions $c=0.7 h$, and for the radius of local subdomains $r_{0}=0.05 \mathrm{~h}$. In all contour graphs, coloration is determined by a constant threshold value, $u_{0}$ for $u$ and $v_{0}$ for $v$, such that in the regions with white colour, $u<u_{0}$ and $v<v_{0}$ while the regions with the concentration $u>u_{0}$ and $v>v_{0}$ are coloured with green. The results confirm that the profiles of the function $v$ are always $180^{\circ}$ out of phase to those of $u$ and therefore only the profiles of $u$ are presented.

\section{Example 1}

Let us analyse the considered problem in the domain $\Omega=[0,1] \times[0,1]$ with 262 nodal points. The parameter $\gamma=100$ is fixed and parameters $(a, b, d)$ are chosen in the Turing space as $a=0.1305, b=0.7695, d=20$. This problem is also considered in $[11,12]$. The time step is $\Delta t=0.005$ and two iterations are used in each time step for consideration of nonlinear terms. Initial conditions read:

$$
\begin{aligned}
& u(x, y, 0)=a+b+u_{p}, u_{p}=10^{-3} \exp \left\{-100\left[(x-1 / 3)^{2}+(y-1 / 2)^{2}\right]\right\}, \\
& v(x, y, 0)=\frac{b}{(a+b)^{2}}
\end{aligned}
$$

where $u_{p}$ is a small perturbation to the homogeneous steady state $\left(u_{0}, v_{0}\right)$. Fig. 1 presents the process of the pattern formation. 


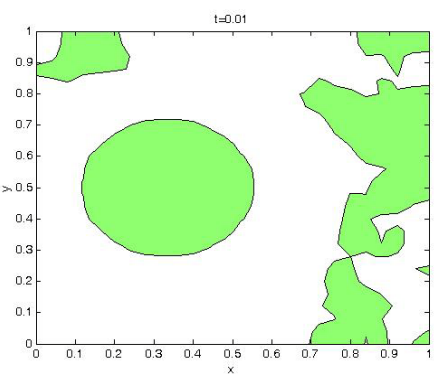

(a) $t=0.01$

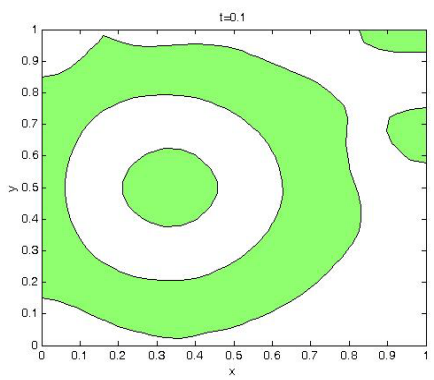

(b) $t=0.1$

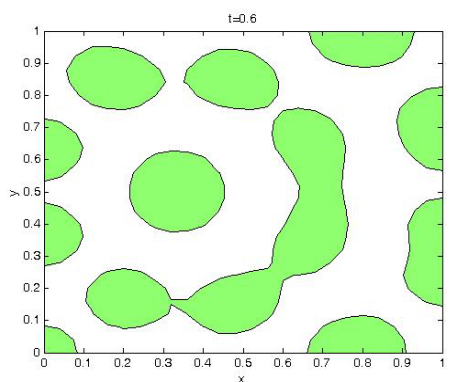

(c) $t=0.6$

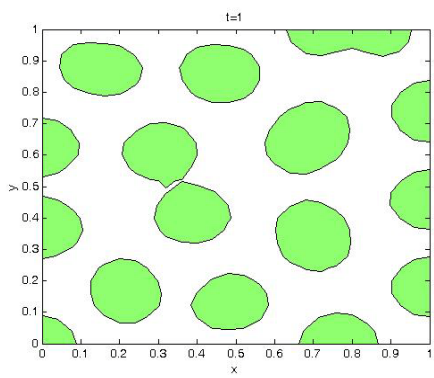

(d) $t=1$

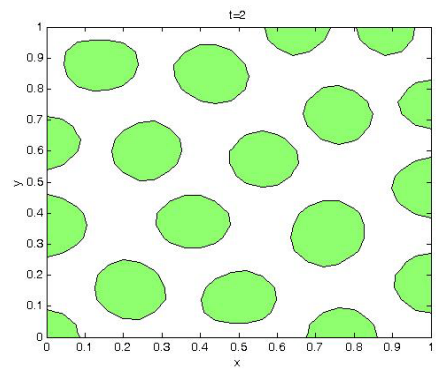

(e) $t=2$

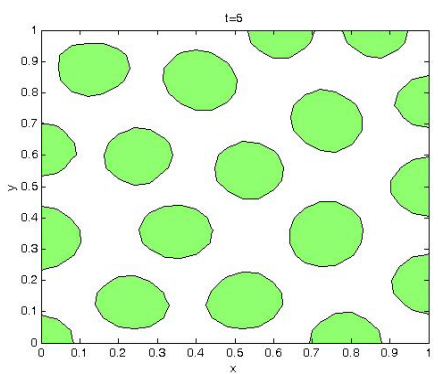

(f) $t=5$

Figure 1: The process of pattern formation with parameters in Example 1.

\section{Example 2}

To see the effect of parameter $\gamma$ on the pattern formation, we fix all parameters as in Ex. 1 and study the model with different values of $\gamma$. The results at the time instant $t=5$ with $\gamma=10,50,75$, and 100 are presented in Fig. 2. The figures on the 1.h.s. are contour plots corresponding to standard colour plots in the r.h.s. It can be seen that increasing the parameter $\gamma$ results in shortening the lengths of allowable patterns (i.e., in increasing the wavenumbers in accordance with Eq. (26)). 

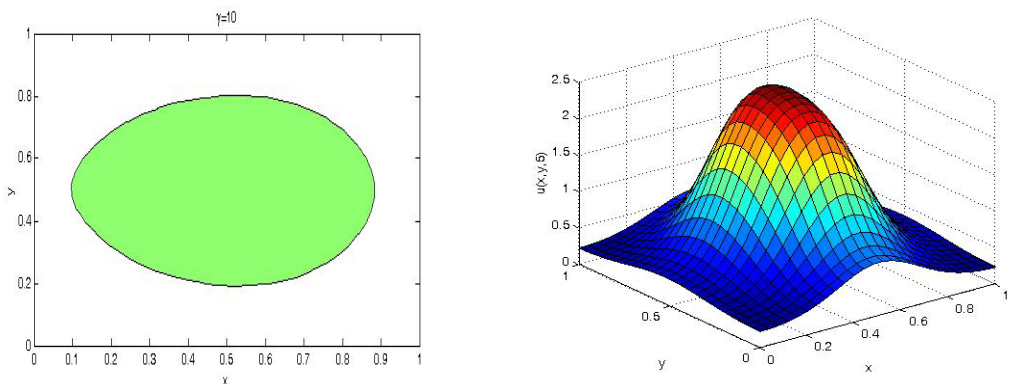

(a) $\gamma=10$
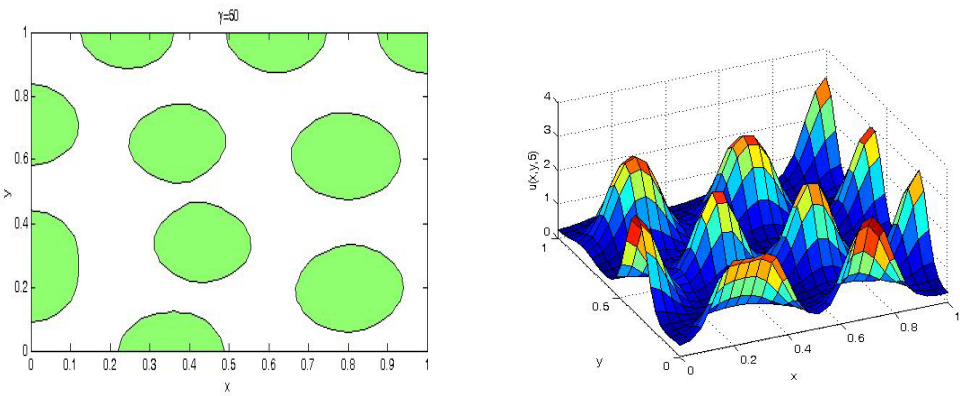

(b) $\gamma=50$
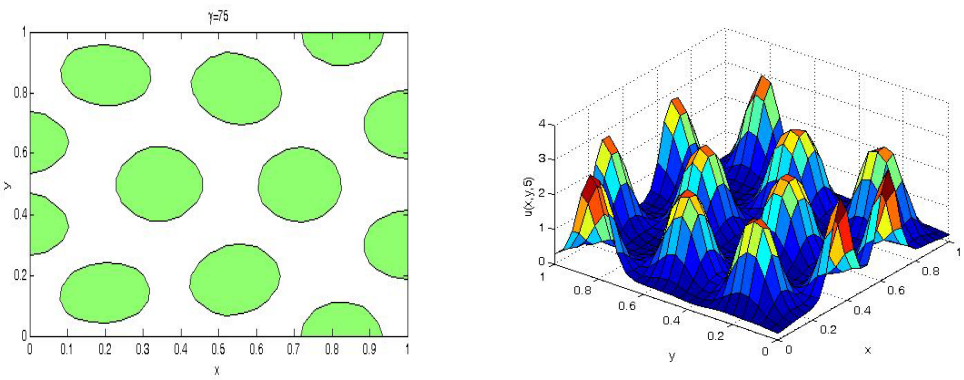

(c) $\gamma=75$
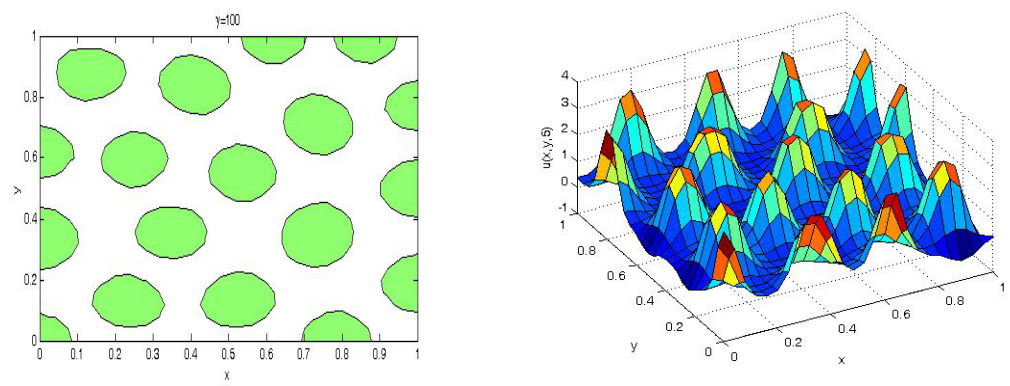

(d) $\gamma=100$

Figure 2: $\quad$ Pattern formation with different values of $\gamma$ at time instant $t=5$. 


\section{Conclusions}

Pattern formations in nonlinear reaction diffusion systems combining local activation with long range inhibition were numerically simulated by the aid of Local Integral Equation method. The weak formulation on local subdomains was developed for solution of initial-boundary value problems for the set of nonlinear governing partial differential equations. Spatial variations of the field variables were discretized by the MLS approximation. Special techniques were proposed to numerically evaluate the integrals appearing in the LIE formulation. A one step finite difference method was adopted for discretizing the time variable. The final system of nonlinear equations was treated iteratively within each time step. In order to verify the accuracy of the proposed method, we considered the Schnakenberg model which is one of the most interesting Turing models in pattern formation. The dependence of pattern shape on the initial and boundary conditions, size and shape of the analysed domain were studied.

\section{Acknowledgement}

This work was partially supported by the Slovak Research and Development Agency under the contract No. APVV-0032-10.

\section{References}

[1] Turing, A., The chemical basis of morphogenesis. Phil. Trans. R. Soc. Lond. B, 237, pp. 37-72, 1952.

[2] Murray, J.D., Mathematical Biology, Springer-Verlag: Berlin, 2003.

[3] Gierer, A., Meinhardt, H., A theory of biological pattern formation. Kybernetik, 12, pp. 30-39, 1972.

[4] Gray, P., Scott, S.K., Autocatalytic reactions in the isothermal, continuous stirred tank reactor. Chemical Engineering Science, 38, pp. 29-43, 1983.

[5] Lengyel, I., Epstein, I.R., Modeling of Turing structures in the chloriteiodidemalonic acid-starch reaction system. Science, 251, pp. 650-652, 1991.

[6] Prigogine, I., Lefever, R., Symmetry breaking instabilities in dissipative systems II. Journal of Chemical Physics, 48, pp. 1665-1700, 1968.

[7] Schnakenberg, J., Simple chemical reaction system with limit cycle behaviour. Journal of Theoretical Biology, 81, pp. 389-400, 1979.

[8] Sel'kov, E.E., Self-oscillations in glycolysis. European Journal of Biochemistry, 4, pp. 79-86, 1968.

[9] Lancaster, P., Salkauskas, K., Surfaces generated by moving least square method. Math. Comput., 37, pp. 141-158, 1981.

[10] Sladek, V., Sladek, J., Local integral equations implemented by MLSapproximation and analytical integrations. Eng. Anal. Bound. Elem., 34, pp. 904-913, 2010. 
[11] Shakeri, F., Dehghan, M., The finite volume spectral element method to solve Turing models in the biological pattern formation. Computers and Mathematics with Applications, 62, pp. 4322-4336, 2011.

[12] Zhu, J., Zhang, Y., Newman, S., Alber, M., Application of discontinuous Galerkin methods for reaction-diffusion systems in developmental biology. J. Sci. Comput., 40, pp. 391-418, 2009. 